\title{
The Zebrafish Breathes New Life into the Study of Tuberculosis
}

\author{
Henna Myllymäki ${ }^{*}$, Carina A. Bäuerlein ${ }^{1}$ and Mika Rämet $t^{1,2,3,4}$ \\ ${ }^{1}$ BioMediTech, University of Tampere, Tampere, Finland, '2Department of Pediatrics, Tampere University Hospital, Tampere, \\ Finland, ${ }^{3}$ Department of Children and Adolescents, Oulu University Hospital, Oulu, Finland, ${ }^{4}$ EEDEGO Research Unit, Medical \\ Research Center Oulu, University of Oulu, Oulu, Finland
}

Tuberculosis (TB) is a global health emergency. Up to one-third of the world's population is infected with Mycobacterium tuberculosis, and the pathogen continues to kill 1.5 million people annually. Currently, the means for preventing, diagnosing, and treating TB are unsatisfactory. One of the main reasons for the poor progress in TB research has been a lack of good animal models to study the latency, dormancy, and reactivation of the disease. Although sophisticated in vitro and in silico methods suitable for TB research are constantly being developed, they cannot reproduce the complete vertebrate immune system and its interplay with pathogens and vaccines. However, the zebrafish has recently emerged as a useful alternative to more traditional models, such as mice, rabbits, guinea pigs, and non-human primates, for studying the complex pathophysiology of

OPEN ACCESS

Edited by:

Jason Paul Gigley,

University of Wyoming, USA

Reviewed by:

Marc S. Dionne,

Imperial College London, UK

Philip Elks,

University of Sheffield, UK

*Correspondence:

Henna Myllymäki

henna.myllymaki@staff.uta.fi

Specialty section:

This article was submitted

to Microbial Immunology,

a section of the journal

Frontiers in Immunology

Received: 29 February 2016

Accepted: 06 May 2016

Published: 19 May 2016

Citation:

Myllymäki $H$, Bäuerlein CA and Rämet M (2016) The Zebrafish

Breathes New Life into the

Study of Tuberculosis.

Front. Immunol. 7:196.

doi: 10.3389/fimmu.2016.00196 a mycobacterial infection. The model is based on the similarity between Mycobacterium marinum - a natural fish pathogen - and $M$. tuberculosis. In both zebrafish larvae and adult fish, an infection with $M$. marinum leads to the formation of macrophage aggregates and granulomas, which resemble the $M$. tuberculosis infections in humans. In this review, we will summarize the current status of the zebrafish model in TB research and highlight the advantages of using zebrafish to dissect mycobacterial virulence strategies as well as the host immune responses elicited against them. In addition, we will discuss the possibilities of using the adult zebrafish model for studying latency, dormancy, and reactivation in a mycobacterial infection.

Keywords: tuberculosis, zebrafish model system, vaccination, Mycobacterium marinum, Mycobacterium tuberculosis, Mycobacterium infections, granuloma, latency

\section{INTRODUCTION}

Tuberculosis (TB) is still the world's second deadliest infectious disease killing 1.5 million people and with an estimated 9.6 million new cases reported to the WHO in 2015 (1). An estimated one-third of the world's population has been exposed to TB. $5-10 \%$ of these latent carriers will eventually develop the active disease (1).

The causative agent of TB, Mycobacterium tuberculosis, spreads through the air (Figure 1A). Alveolar macrophages phagocytose the inhaled mycobacteria and transport them into the lung tissues (2). A cascade of pro- and anti-inflammatory signaling leads to the recruitment and accumulation of additional macrophages and other leukocytes in the pulmonary tissues. Eventually, the formation of granulomas, the hallmark of pathological TB, is initiated. The granuloma is a heterogeneous, but well-organized, and dynamic accumulation of immune cells, including 
A
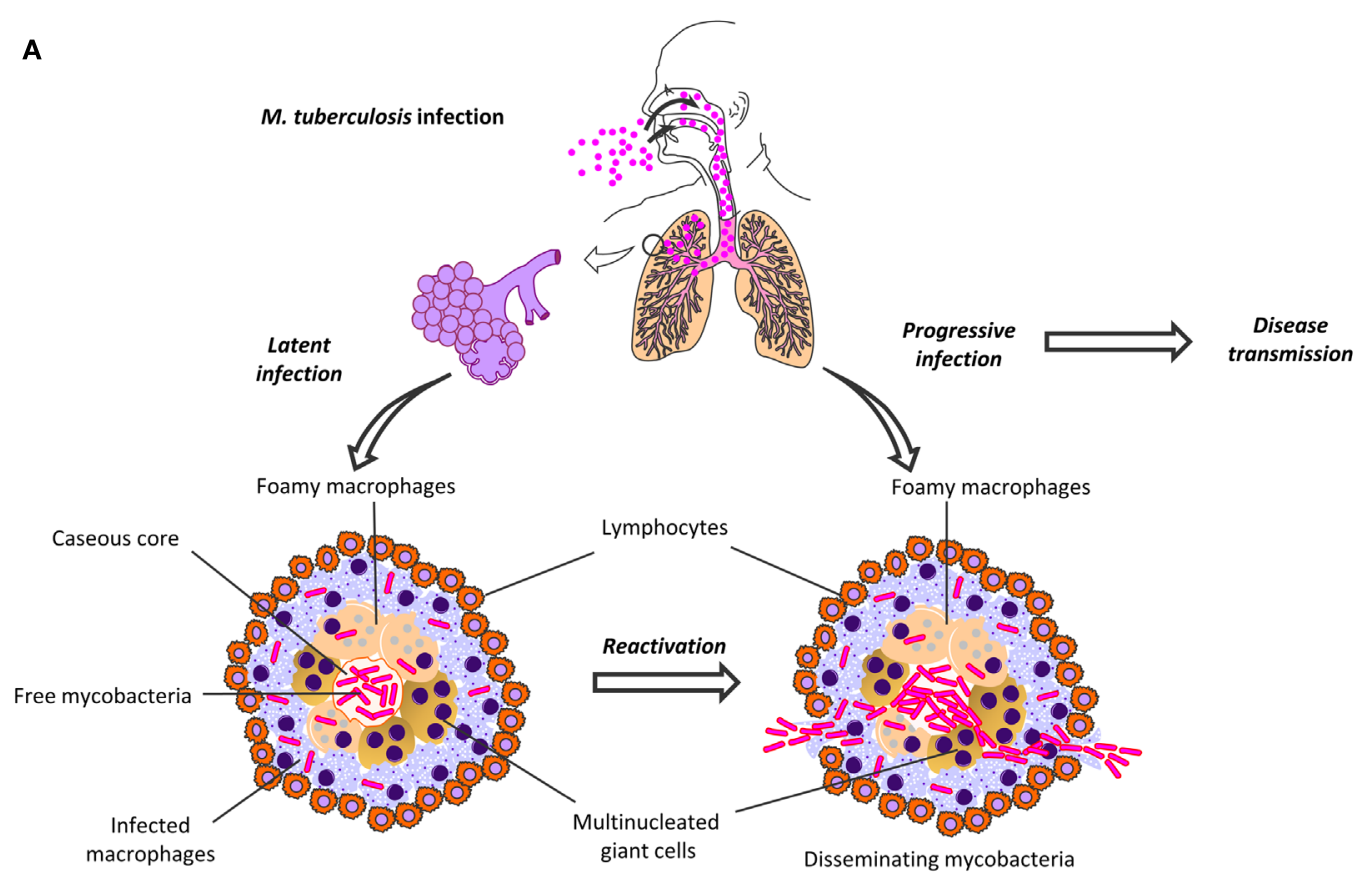

B

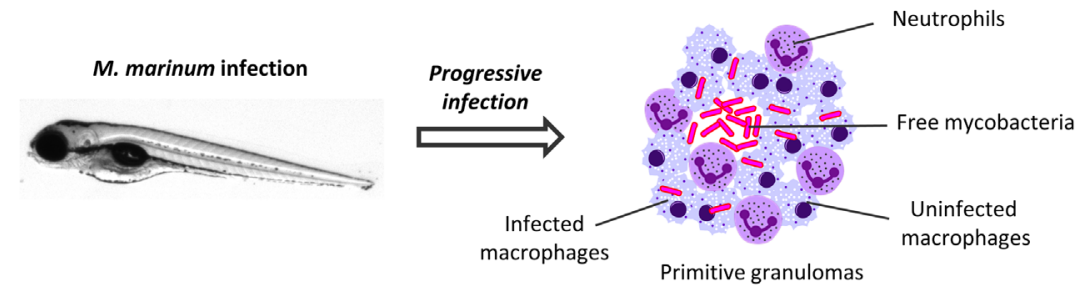

C
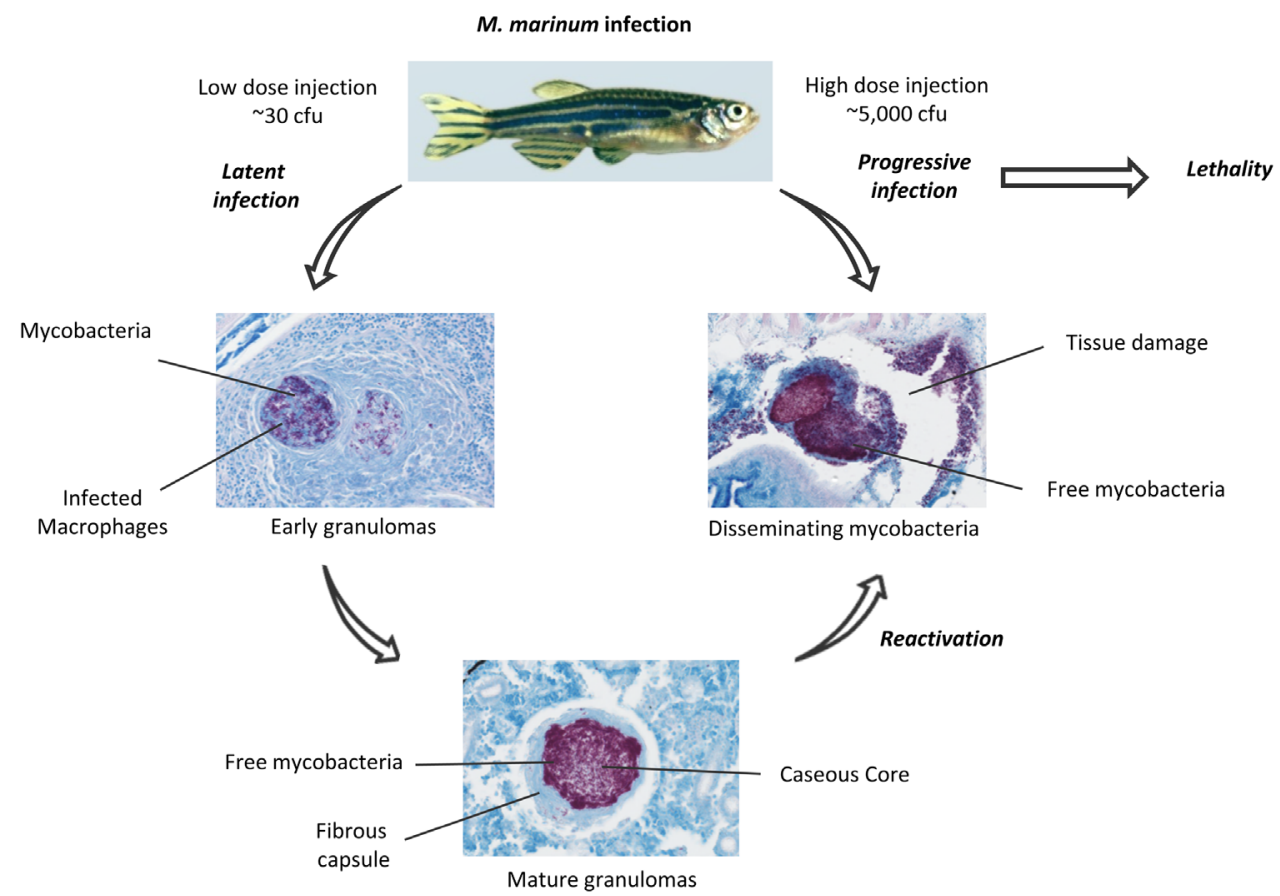

FIGURE 1 | Granuloma formation in human Mycobacterium tuberculosis infection and in zebrafish $M$. marinum infection 


\section{FIGURE $1 \mid$ Continued}

(A) M. tuberculosis spreads as an aerosol, and first infects alveolar macrophages. In most individuals, the infection is maintained in a latent, subclinical state, which is characterized by the formation of granulomas (left). The mature granulomas have a caseous, necrotic core, surrounded by infected macrophages and lymphocytes. Upon reactivation, the granulomas are disrupted, causing cavities in the lungs. The mycobacteria escape from the granulomas and are disseminated in cough droplets, which facilitates the transmission of the disease. (B) Upon infection with M. marinum, the granulomas in the zebrafish embryo develop within a few days and mainly consist of infected and uninfected macrophages and recruited neutrophils. (C) Depending on the bacterial dose, the adult zebrafish M. marinum infection can lead to a latent or an active, progressive disease. A latent infection is characterized by the formation of granulomas in various organs. As the early granulomas mature, their inner parts become caseous and surrounded by a fibrous wall. The zebrafish with a latent infection remain asymptomatic. A (re) activated, progressive mycobacterial infection is characterized by the disruption of the granulomatous structures, rapid replication and dissemination of mycobacteria and profound tissue damage. Eventually, a progressive mycobacterial disease will lead to death in most fish. The zebrafish granulomas were visualized with Ziehl-Neelsen staining, mycobacteria are seen as purple rods. cfu, colony-forming unit.

TABLE 1 | Characteristics of a mycobacterial infection in humans, adult zebrafish, and zebrafish embryos.

\begin{tabular}{|c|c|c|c|c|}
\hline & Human & Adult zebrafish (>3 months) & Zebrafish embryo (<6 days) & Reference \\
\hline Immune system & Innate and adaptive & Innate and adaptive & Innate only & $(6-8)$ \\
\hline Mycobacterial pathogen & $\begin{array}{l}\text { Mycobacterium tuberculosis and atypical } \\
\text { mycobacteria including Mycobacterium marinum }\end{array}$ & Mycobacterium marinum & Mycobacterium marinum & $(9-11)$ \\
\hline Natural susceptibility & Yes & Yes & Yes & $(9,10,12)$ \\
\hline \multirow{4}{*}{$\begin{array}{l}\text { Infection route and } \\
\text { infectious dose }\end{array}$} & \multicolumn{4}{|c|}{ Natural infection } \\
\hline & Airways $\mathrm{ID}_{50}<10$ bacilli & Digestive tract & $?$ & $(9,10,12)$ \\
\hline & \multicolumn{4}{|c|}{ Experimental infection } \\
\hline & $\mathrm{N} / \mathrm{A}$ & $\begin{array}{l}\text { Multiple injection techniques, } \\
\text { intraperitoneal injection is the most } \\
\text { commonly used; }<30-10,000 \mathrm{cfu}\end{array}$ & $\begin{array}{l}\text { Multiple injection techniques, } \\
\text { caudal injection is the most } \\
\text { commonly used; <10->300 cfu }\end{array}$ & $(8,13-15)$ \\
\hline \multirow[t]{3}{*}{ Infection phases } & Acute & Acute & \multirow[t]{3}{*}{ Progressive? } & \multirow[t]{3}{*}{$(10,13-17)$} \\
\hline & Latent & Latent & & \\
\hline & Reactivation & Reactivation & & \\
\hline \multirow[t]{3}{*}{ Granuloma types } & Early & Early & \multirow[t]{3}{*}{ Primitive? } & \multirow{3}{*}{$\begin{array}{c}(4,5 \\
13-18)\end{array}$} \\
\hline & Fibrous & Fibrous & & \\
\hline & Necrotic & Necrotic & & \\
\hline \multirow{6}{*}{$\begin{array}{l}\text { Cell types involved in } \\
\text { granuloma formation }\end{array}$} & Macrophages & Macrophages & Macrophages & \multirow{6}{*}{$\begin{array}{c}(4,5 \\
13-18)\end{array}$} \\
\hline & Neutrophils & Neutrophils & Neutrophils & \\
\hline & Dendritic cells & Dendritic cells & Epithelial cells & \\
\hline & Lymphocytes ( $T$ cells, B cells, and NK cells) & Lymphocytes (T cells and B cells) & & \\
\hline & Fibroblasts & Epithelial cells & & \\
\hline & Epithelial cells & & & \\
\hline
\end{tabular}

blood-derived infected and uninfected macrophages, foamy macrophages, and epithelioid cells (3). The inner cell mass is usually surrounded by a ring of leukocytes and fibroblasts (4) (Figure 1A; Table 1). The localization and control of the bacteria and the restriction of the immune response to a defined area are generally regarded as the main functions of granulomas (5).

Mycobacteria can persist asymptomatic within the granuloma for decades. However, a dysregulation of the immune system can reactivate the mycobacteria, which leads to disease progression (secondary TB) (2) (Figure 1A) Primary TB mainly occurs in children, who are at the highest risk for TB meningitis and a disseminated form of the disease (19).

Efforts to eradicate TB are obstructed by the lack of unambiguous diagnostic tools, the lengthy antibiotic treatments required for curation, the growing problem of multi-drug-resistant bacteria, and the poor protection provided by the Bacillus Calmette-Guérin (BCG), the only vaccine available (1). As a live vaccine, BCG imposes a risk of a disseminated infection in immunocompromised patients $(1,20,21)$. Thus, there is a need to develop new effective drugs and vaccines against TB. For this purpose, relevant animal models are essential. The most commonly used animal models in TB research are mice, guinea pigs, and non-human primates (NHP), all of which have their limitations related to either space, costs, ethical aspects, or their ability to replicate the human disease pathology $(22,23)$. Recently, the zebrafish-M. marinum model has gained popularity as a natural pathogen-host system that closely recapitulates the pathology of human TB (Table 1) $(13,23)$. The infection model and its applications are discussed in more detail below.

\section{THE ZEBRAFISH-MYCOBACTERIUM MARINUM INFECTION MODEL}

Mycobacterium marinum, the causative agent of fish mycobacteriosis, is a close relative of M. tuberculosis (24). M. marinum 
spreads via water, and it also occasionally infects humans, but the infection is usually limited to the skin (fish tank granuloma) (11). Thus, M. marinum is safer to work with and has a shorter replication time than $M$. tuberculosis $(9,23)$. Similar to human TB, fish mycobacteriosis displays an acute and chronic form and the subsequently formed granulomas also resemble the lesions caused by M. tuberculosis [Figure 1 (13-15)]. Both bacteria are able to survive and replicate within macrophages $(23,25)$. In a laboratory setting, the zebrafish is an advantageous choice as a host organism for M. marinum for several reasons: multiple infection techniques can be used for both zebrafish embryos and adults (26); for a review, see Ref. (8, 27-29) (Figures 1B,C). The transparency of the embryos allows the use of sophisticated in vivo real-time imaging techniques, including multiple leukocyte and macrophage fluorescent reporter lines (30-32), and several techniques for genetic manipulation (6, 33-35). Moreover, zebrafish are small in size, and produce numerous offspring, making them also suitable for large-scale screening studies, including drug screens (6). Despite the anatomical differences between fish and mammals, the zebrafish is a vertebrate model with an innate and adaptive immunity consisting of the same primary components as present in humans $(6,36,37)$. As zebrafish lack lymph nodes, immune cells mainly develop and perform their functions in the spleen, the kidney, and the thymus $(6,38,39)$. Zebrafish embryos rely solely on innate immunity. In the embryos, functional macrophages and neutrophils emerge 1 and 2 days post-fertilization (dpf), respectively, while lymphocytes start developing after $4 \mathrm{dpf}$ and the adaptive immunity becomes fully functional at 4 weeks post-fertilization (wpf) (7). This facilitates studying the function of the innate and adaptive immune system, as well as different cell types, separately $(8,33)$.

\section{HOST-MYCOBACTERIUM INTERACTIONS - INSIGHTS INTO EARLY INFECTION EVENTS FROM ZEBRAFISH LARVAE}

Zebrafish larvae have been especially useful in elucidating the role of macrophages and the strategies the phagocytosed mycobacteria use to suppress phagosomal maturation, apoptosis, and the antibacterial innate immune response $(8,25,40)$. Scavenger receptors of different classes have been studied in the context of the phagocytosis of mycobacteria. For example, Marco binds the glycolipid trehalose 6,69-dimycolate on the mycobacterial cell wall and affects the regulation of the subsequent proinflammatory response (41). CD36 also appears to be involved in mycobacterial control, though its role and the regulation of its expression seem quite complicated (42). Following phagocytosis, Toll-like receptor (TLR) signaling via MyD88 is needed for resistance against an early mycobacterial infection $(43,44)$. For example, the activation of the TLR signaling leads to the production of antibacterial molecules by macrophages, such as the

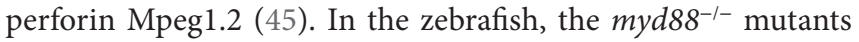
have been a useful tool in elucidating the role of TLR signaling in mycobacterial resistance $(46,47)$.
Toll-like receptor signaling is involved in the initiation of the production of reactive oxygen and nitrogen species (ROS and RNS, respectively). In the zebrafish, early stabilization of the transcription factor hypoxia-inducible factor $1 \alpha$ (Hif- $1 \alpha)$ in neutrophils limits bacterial growth by inducing iNOS, which in turn leads to increased protein nitrosylation (48). Later in the course of the infection, this response is decreased in the developing granulomas by a mechanism independent of the early secretory antigenic target 6 system 1/region of difference 1 (ESX-1/RD1) virulence locus (49).

As a mean to counteract the mycobacterial evasion strategies, the host can induce autophagy, a process which enables cells to digest their cytoplasmic contents, including microorganisms and membranous structures, in lysosomes (50). This is dependent on TLR signaling and Myd88, which are linked via the DNA damage-regulated autophagy modulator DRAM1. DRAM1 is needed for the formation of autophagosomes and for their fusion with lysosomes, while a DRAM1 deficiency leads to defects in maintaining the mycobacteria inside vesicles in macrophages and in the control of mycobacterial growth (51), Again, zebrafish larvae provide feasible tools for observing the events of autophagy in vivo and in real-time utilizing both light and electron microscopy, including the GFP-Lc3-transgenic line, for the visualization of autophagosomal structures (52).

\section{ZEBRAFISH LARVAE MODEL CHALLENGE OLD DOGMAS IN MYCOBACTERIAL RESISTANCE}

After a successful infection by mycobacteria, granulomas are seeded. The granulomas in zebrafish embryos mainly consist of aggregated macrophages, intra- and extracellular mycobacteria, together with recruited neutrophils, and form within a few days post-infection $(18,53)$; for a review, see Ref. (54). Despite their rather primitive structure, the embryonic zebrafish granulomas provide a physiological model for studying cellular processes affecting mycobacterial infections, such as the generation of hypoxia and angiogenesis. Injection of mycobacteria into the caudal vein results in the development of non-hypoxic granulomas in the richly vascularized area of the caudal hematopoietic tissue (48). In contrast, granulomas resulting from a trunk infection reside in a sparsely vascularized area and can become hypoxic and induce vascularization (55).

Importantly, studies on early granulomas in zebrafish larvae have challenged some of the old dogmas. In general, granulomas have long been considered a protection mechanism elicited by the host. However, recent evidence from zebrafish embryos suggests that $M$. marinum uses the macrophages and granulomas for its own expansion and dissemination $(16,17)$. The bacteria can recruit new, uninfected macrophages to the granuloma site using the RD1 locus (16). The arriving macrophages phagocytize infected, dead cells and thus contribute to the spreading of the bacteria. The recruitment of new macrophages is enhanced by the bacteria by ESAT-6-mediated production of matrix metalloproteinase 9 (17). Mycobacteria also need the RD1 locus for escaping from the phagosomes into the cytoplasm of infected macrophages both in 
human cells and in zebrafish $(51,56)$. Additionally, mycobacteria use cell surface lipids to mask pathogen-associated molecular patterns, hence selectively infect permissive macrophages and avoid microbicidal ones (57). Thus, granulomas present a combat zone for the host immune system and the bacterial response, rather than purely a protection mechanism of the host to prevent the spread of bacteria (4).

In addition to basic research, the zebrafish larvae provide a feasible tool for early-stage drug development and large-scale screens $(33,58,59)$. Two major, and related, issues in improving the TB drug development are the lengthiness of the curative treatments and the increasing emergence of drug-resistant bacteria (1). Discoveries made in zebrafish larvae revealed that intracellular mycobacteria use their efflux pumps to acquire a tolerance against the antibiotics commonly used to treat human $\mathrm{TB}$, which allows the bacteria to persist and replicate in the cytosol. M. tuberculosis uses the same mechanism (60). However, this tolerance was reversed by an efflux pump inhibitor, such as verapamil, which can therefore reduce the tolerance to antibiotics and thereby shorten treatment times (61). These results prove that the zebrafish larval model can replicate the function of antitubercular compounds (60). In addition, angiogenesis has been shown to be important for granuloma formation. Therefore, targeting vascularization, for example, by inhibiting vascular endothelial growth factor receptor (VEGFR) signaling could provide a means to target mycobacterial infections and inhibit mycobacterial dissemination, resembling the strategy used in cancer therapies (55).

On the host's side, cytokines and their respective receptors play an important role in protection against mycobacteria. For example, the signaling axis mediated by chemokine CXC-motive containing receptor 3 (CXCR3) has been implicated in mycobacterial spreading and could thus provide a therapeutic target (62). In addition, in both humans and zebrafish larvae the leukotriene A4 hydrolase (LTA4H) locus controls pro- and anti-inflammatory mediators that in turn control the expression of TNF (63). While TNF is required for the host response against mycobacteria, its excess renders the host more susceptible to an infection, highlighting the importance of a balanced response (64). Thus, the zebrafish can elucidate the pathways controlling the host immune responses, and this information can be further applied to targeting these pathways with new drugs and developing host-directed therapies $(8,63-66)$. In addition, further investigation into the evasion strategies that mycobacteria use to interfere with the host defense mechanisms can potentially lead to the discovery of novel drug targets to combat mycobacterial diseases $(49,67)$.

\section{MYCOBACTERIAL LATENCY VS. ADAPTIVE IMMUNITY - THE ADULT ZEBRAFISH AS A MODEL FOR TB}

One of the main advantages of the zebrafish-M. marinum model may lie in granuloma formation, which has not been easy to reproduce in the traditional model animals (22). In the adult zebrafish, however, the histology of the mature granulomas resembles those seen in human TB with their caseous, necrotic core surrounded by leukocytes and epithelial cells $(4,17)$. Granulomas are found in various organs such as the pancreas, gonads, spleen, and liver several weeks post-infection (Figure 1C) (13-15).

The latency of TB is another aspect that has been difficult to replicate experimentally (22). As this is also challenging to study in humans, our knowledge of the required immunological mechanisms for the control of a mycobacterial infection at its different stages remains limited (17). However, this could be improved by studying the zebrafish model, as like humans, adult zebrafish develop a latent, non-progressive disease with dormant bacteria residing within well-structured granulomas, and the fish remain asymptomatic $(13,14)$. Moreover, reactivation of the bacteria can occur spontaneously, or can be induced experimentally by an immune deficiency, such as exposure to $\gamma$-irradiation. In either case, reactivation will lead to the active spreading of the bacteria and the development of symptoms similar to an active infection as well as high lethality, much as in human TB (13).

Although the early cytokine response mediated by the innate immunity plays an important role in determining whether a mycobacterial infection leads to an acute or latent disease, the adaptive immunity is also required to control the bacteria. This is seen in the adult $\mathrm{rag}^{-1-}$ zebrafish that are devoid of lymphocytes. The mutant fish are unable to generate a latent state of the infection and are, therefore, hypersusceptible to $M$. marinum $(13,14,68)$. So far, the significance of different lymphocyte subsets has not been extensively studied in the zebrafish mycobacterial infection. Nevertheless, Th1 as well as Th2 cells seem to be involved in the effective control of mycobacterial infections $(69,70)$. Interestingly, a novel Th2-like subset of cells capable of inhibiting the growth of $M$. tuberculosis has been found in human TB patients. This observation challenges the old idea that only Th1 cells are important for mycobacterial control (71). Genetic differences in the mycobacterial strains also seem to affect their virulence, for example, strains isolated from infected humans more commonly causing an acute disease, and isolates from poikilothermic species causing a chronic infection in the zebrafish (72).

While the zebrafish larvae provide a feasible tool for screening for drugs against TB, the adult zebrafish appears to be a promising model for early vaccine development. The zebrafish can be partially protected against mycobacteriosis by BCG $(68,73)$ or attenuated M. marinum (74), suggesting the use of adult zebrafish as a model for studying the feasibility of conserved mycobacterial antigens as vaccines. For example, the RD1 virulence locus and the ESX-1 secretion system, which are absent from BCG, have been shown to be important for virulence in M. tuberculosis. In $M$. marinum, $\mathrm{RD} 1$ is also is also required for granuloma formation in both larvae and adult fish $(14,49,75,76)$. Indeed, the antigens in this region do show some potential as targets for vaccines in the zebrafish as well as in other models $(68,73,77-79)$. The zebrafish can also provide a feasible model for searching for the most effective antigen combinations and for studying different vaccination strategies. For instance, a DNA-based vaccine consisting of three mycobacterial antigens (Ag85, ESAT-6, and CFP10), which has also been studied in other TB models, confers protection against mycobacteria in zebrafish $(68,80-82)$. Furthermore, the effect of BCG can be boosted by a DNA vaccine $(68,73,80-82)$. Adult zebrafish could therefore be used for developing vaccines for 
both replacing and boosting BCG, as well as for studying the immunological correlates required for protection.

\section{DISCUSSION}

The lack of an animal model that recapitulates the human disease stages and pathology has in part hampered the development of new drugs, vaccines, and diagnostic tools against TB (22). Even though the mammalian animal models mostly used for TB research, namely, mice, rabbits, and guinea pigs, do develop granulomatous structures, only primates are a natural host for M. tuberculosis and show true latency and reactivation (83). However, the use of primates as laboratory animals is difficult in terms of ethical and economic issues as well as space limitations. When drug and vaccine development is considered, a natural host-pathogen pair is likely to be a more reliable model. Moreover, choosing to work with (zebra)fish might have an additional practical advantage: as mycobacterial infections are able to cause epidemics in fish farms, aquariums, and zebrafish facilities $(9,84)$, vaccinating fish against $M$. marinum is of potential economic and ecological relevance. This has been studied to some extent, for example, in the striped bass with the Ag85A DNA vaccine (85) and in the Japanese flounder with BCG (86). Thus, the results obtained in human biomedical research and veterinary studies could potentially augment each other.

The zebrafish- $M$. marinum infection exhibits essentially the same disease phases as those seen in human TB, including latency and reactivation - either spontaneously or following immunosuppression $(13,14)$. This might have important implications as the different disease phases probably also represent on the one hand different strategies of bacterial adaptation, and on the other hand, different stages of the host immune response (87). Since these aspects can be replicated in the zebrafish infection, the model should facilitate a more detailed dissection of both the effective (and deleterious) immune responses and the bacterial counter strategies in each stage of the infection. The zebrafish model can also be used to complement the human patient data in the identification of reliable biomarkers for the diagnosis of the different stages of TB (88).

Besides biomarkers, new drugs and vaccines are needed to combat TB. For this, a better knowledge concerning the correlates of a protective immune response is essential (89). The HI virus

\section{REFERENCES}

1. World Health Organization. Global Tuberculosis Report 2015. (2015). Available from: http://www.who.int/tb/publications/global_report/en/

2. Frieden TR, Sterling TR, Munsiff SS, Watt CJ, Dye C. Tuberculosis. Lancet (2003) 362:887-99. doi:10.1016/S0140-6736(03)14333-4

3. Adams DO. The structure of mononuclear phagocytes differentiating in vivo. I. Sequential fine and histologic studies of the effect of Bacillus CalmetteGuerin (BCG). Am J Pathol (1974) 76:17-48.

4. Ramakrishnan L. Revisiting the role of the granuloma in tuberculosis. Nat Rev Immunol (2012) 12:352-66. doi:10.1038/nri3211

5. Adams DO. The granulomatous inflammatory response. A review. Am J Pathol (1976) 84(1):164-92. attacks $\mathrm{CD}^{+} \mathrm{T}$ cells, and a co-infection renders the patients highly susceptible to TB. Therefore, it seems that $\mathrm{CD}^{+} \mathrm{T}$ cells are important for the host response (90). This does not mean, however, that the study of other cell types should be neglected, as they too can reveal new immunological mechanisms (70, 71). Moreover, deficiencies in the IFN- $\gamma$ signaling axis lead to hypersusceptibility toward TB, and thus IFN- $\gamma$ expression has been associated with protection against the disease (90). However, despite inducing high levels of IFN- $\gamma$ production, a promising vaccine candidate, MVA85A, failed recently to enhance protection in an efficacy trial (91). This suggests that it is unlikely that a single immunological factor could predict the course of a TB infection $(90,92)$. Thus, there are still gaps in our knowledge of how an effective host defense against TB is elicited, and relevant animal models are needed to fill in the missing information. Furthermore, once the picture of protection mechanisms is more complete, the animal models can aid us in translating this information into the benefit of clinical medicine. We believe the zebrafish will be an important player in fulfilling both of these tasks.

\section{AUTHOR CONTRIBUTIONS}

All authors contributed to planning and writing the manuscript and designing of the figures.

\section{ACKNOWLEDGMENTS}

We thank Dr. Helen Cooper for revising the language of the manuscript and MSc Mirja Niskanen for technical help with histology and MSc Meri Uusi-Mäkelä for help with the figure.

\section{FUNDING}

This work was supported by the Foundation of the Finnish Anti-Tuberculosis Association (HM), the Tampere Tuberculosis Foundation (HM and MR), the Finnish Academy (MR) (grant number on 277495), the Sigrid Juselius Foundation (MR), the Jane and Aatos Erkko Foundation (MR), the Competitive State Research Financing of the Expert Responsibility Area of Tampere University Hospital (MR), and Competitive State Research Financing of the Expert Responsibility area of Oulu University Hospital (MR).

6. Renshaw SA, Trede NS. A model 450 million years in the making: zebrafish and vertebrate immunity. Dis Model Mech (2012) 5:38-47. doi:10.1242/ dmm.007138

7. Langenau DM, Ferrando AA, Traver D, Kutok JL, Hezel JP, Kanki JP, et al. In vivo tracking of $\mathrm{T}$ cell development, ablation, and engraftment in transgenic zebrafish. Proc Natl Acad Sci U S A (2004) 101:7369-74. doi:10.1073/ pnas.0402248101

8. Meijer AH, Spaink HP. Host-pathogen interactions made transparent with the zebrafish model. Curr Drug Targets (2011) 12:1000-17. doi:10.2174/138945011795677809

9. Decostere A, Hermans K, Haesebrouck F. Piscine mycobacteriosis: a literature review covering the agent and the disease it causes in fish and humans. Vet Microbiol (2004) 99:159-66. doi:10.1016/j.vetmic.2003.07.011 
10. O'Garra A, Redford PS, McNab FW, Bloom CI, Wilkinson RJ, Berry MP. The immune response in tuberculosis. Annu Rev Immunol (2013) 31:475-527. doi:10.1146/annurev-immunol-032712-095939

11. Linell F, Norden A. Mycobacterium balnei, a new acid-fast bacillus occurring in swimming pools and capable of producing skin lesions in humans. Acta Tuberc Scand Suppl (1954) 33:1-84.

12. Harriff MJ, Bermudez LE, Kent ML. Experimental exposure of zebrafish, Danio rerio (Hamilton), to Mycobacterium marinum and Mycobacterium peregrinum reveals the gastrointestinal tract as the primary route of infection: a potential model for environmental mycobacterial infection. JFish Dis (2007) 30:587-600. doi:10.1111/j.1365-2761. 2007.00839.x

13. Parikka M, Hammaren MM, Harjula SK, Halfpenny NJ, Oksanen KE, Lahtinen MJ, et al. Mycobacterium marinum causes a latent infection that can be reactivated by gamma irradiation in adult zebrafish. PLoS Pathog (2012) 8:e1002944. doi:10.1371/journal.ppat.1002944

14. Swaim LE, Connolly LE, Volkman HE, Humbert O, Born DE, Ramakrishnan L. Mycobacterium marinum infection of adult zebrafish causes caseating granulomatous tuberculosis and is moderated by adaptive immunity. Infect Immun (2006) 74:6108-17. doi:10.1128/IAI.00887-06

15. Prouty MG, Correa NE, Barker LP, Jagadeeswaran P, Klose KE. ZebrafishMycobacterium marinum model for mycobacterial pathogenesis. FEMS Microbiol Lett (2003) 225:177-82. doi:10.1016/S0378-1097(03)00446-4

16. Davis JM, Ramakrishnan L. The role of the granuloma in expansion and dissemination of early tuberculous infection. Cell (2009) 136:37-49. doi:10.1016/j.cell.2008.11.014

17. Volkman HE, Pozos TC, Zheng J, Davis JM, Rawls JF, Ramakrishnan L. Tuberculous granuloma induction via interaction of a bacterial secreted protein with host epithelium. Science (2010) 327:466-9. doi:10.1126/ science. 1179663

18. Davis JM, Clay H, Lewis JL, Ghori N, Herbomel P, Ramakrishnan L. Real-time visualization of mycobacterium-macrophage interactions leading to initiation of granuloma formation in zebrafish embryos. Immunity (2002) 17:693-702. doi:10.1016/S1074-7613(02)00475-2

19. Marais BJ, Gie RP, Schaaf HS, Beyers N, Donald PR, Starke JR. Childhood pulmonary tuberculosis: old wisdom and new challenges. Am J Respir Crit Care Med (2006) 173:1078-90. doi:10.1164/rccm.200511-1809SO

20. Roy A, Eisenhut M, Harris RJ, Rodrigues LC, Sridhar S, Habermann S, et al. Effect of BCG vaccination against Mycobacterium tuberculosis infection in children: systematic review and meta-analysis. BMJ (2014) 349:g4643. doi:10.1136/bmj.g4643

21. Mak TK, Hesseling AC, Hussey GD, Cotton MF. Making BCG vaccination programmes safer in the HIV era. Lancet (2008) 372:786-7. doi:10.1016/ S0140-6736(08)61318-5

22. Myllymaki H, Niskanen M, Oksanen KE, Ramet M. Animal models in tuberculosis research - where is the beef? Expert Opin Drug Discov (2015) 10:871-83. doi:10.1517/17460441.2015.1049529

23. Tobin DM, Ramakrishnan L. Comparative pathogenesis of Mycobacterium marinum and Mycobacterium tuberculosis. Cell Microbiol (2008) 10:1027-39. doi:10.1111/j.1462-5822.2008.01133.x

24. Stinear TP, Seemann T, Harrison PF, Jenkin GA, Davies JK, Johnson PD, et al. Insights from the complete genome sequence of Mycobacterium marinum on the evolution of Mycobacterium tuberculosis. Genome Res (2008) 18:729-41. doi:10.1101/gr.075069.107

25. Barker LP, George KM, Falkow S, Small PL. Differential trafficking of live and dead Mycobacterium marinum organisms in macrophages. Infect Immun (1997) 65:1497-504.

26. Benard EL, van der Sar AM, Ellett F, Lieschke GJ, Spaink HP, Meijer AH. Infection of zebrafish embryos with intracellular bacterial pathogens. J Vis Exp (2012) 61:e3781. doi:10.3791/3781

27. Meijer AH. Protection and pathology in TB: learning from the zebrafish model. Semin Immunopathol (2016) 38(2):261-73. doi:10.1007/s00281-015-0522-4

28. van Leeuwen LM, van der Sar AM, Bitter W. Animal models of tuberculosis: zebrafish. Cold Spring Harb Perspect Med (2014) 5:a018580. doi:10.1101/ cshperspect.a018580

29. Cronan MR, Tobin DM. Fit for consumption: zebrafish as a model for tuberculosis. Dis Model Mech (2014) 7:777-84. doi:10.1242/dmm.016089

30. HallC, Flores MV, Crosier K, Crosier P. Live cell imaging of zebrafish leukocytes. Methods Mol Biol (2009) 546:255-71. doi:10.1007/978-1-60327-977-2_16
31. Renshaw SA, Loynes CA, Trushell DM, Elworthy S, Ingham PW, Whyte MK. A transgenic zebrafish model of neutrophilic inflammation. Blood (2006) 108:3976-8. doi:10.1182/blood-2006-05-024075

32. Wittamer V, Bertrand JY, Gutschow PW, Traver D. Characterization of the mononuclear phagocyte system in zebrafish. Blood (2011) 117:7126-35. doi:10.1182/blood-2010-11-321448

33. Lohi O, Parikka M, Rämet M. The zebrafish as a model for paediatric diseases. Acta Paediatr (2013) 102:104-10. doi:10.1111/j.1651-2227.2012.02835.x

34. Hwang WY, Fu Y, Reyon D, Maeder ML, Tsai SQ, Sander JD, et al. Efficient genome editing in zebrafish using a CRISPR-Cas system. Nat Biotechnol (2013) 31:227-9. doi:10.1038/nbt.2501

35. Varshney GK, Sood R, Burgess SM. Understanding and editing the zebrafish genome. Adv Genet (2015) 92:1-52. doi:10.1016/bs.adgen.2015.09.002

36. Traver D, Herbomel P, Patton EE, Murphey RD, Yoder JA, Litman GW, et al. The zebrafish as a model organism to study development of the immune system. Adv Immunol (2003) 81:253-330.

37. Traver D, Paw BH, Poss KD, Penberthy WT, Lin S, Zon LI. Transplantation and in vivo imaging of multilineage engraftment in zebrafish bloodless mutants. Nat Immunol (2003) 4:1238-46. doi:10.1038/ni1007

38. Kissa K, Murayama E, Zapata A, Cortes A, Perret E, Machu C, et al. Live imaging of emerging hematopoietic stem cells and early thymus colonization. Blood (2008) 111:1147-56. doi:10.1182/blood-2007-07-099499

39. Lugo-Villarino G, Balla KM, Stachura DL, Banuelos K, Werneck MB, Traver D. Identification of dendritic antigen-presenting cells in the zebrafish. Proc Natl Acad Sci U S A (2010) 107:15850-5. doi:10.1073/pnas.1000494107

40. Koul A, Herget T, Klebl B, Ullrich A. Interplay between mycobacteria and host signalling pathways. Nat Rev Microbiol (2004) 2:189-202. doi:10.1038/ nrmicro840

41. Benard EL, Roobol SJ, Spaink HP, Meijer AH. Phagocytosis of mycobacteria by zebrafish macrophages is dependent on the scavenger receptor Marco, a key control factor of pro-inflammatory signalling. Dev Comp Immunol (2014) 47:223-33. doi:10.1016/j.dci.2014.07.022

42. Fink IR, Benard EL, Hermsen T, Meijer AH, Forlenza M, Wiegertjes GF. Molecular and functional characterization of the scavenger receptor CD36 in zebrafish and common carp. Mol Immunol (2015) 63:381-93. doi:10.1016/j. molimm.2014.09.010

43. Velez DR, Wejse C, Stryjewski ME, Abbate E, Hulme WF, Myers JL, et al. Variants in Toll-like receptors 2 and 9 influence susceptibility to pulmonary tuberculosis in Caucasians, African-Americans, and West Africans. Hum Genet (2010) 127:65-73. doi:10.1007/s00439-009-0741-7

44. Kleinnijenhuis J, Oosting M, Joosten LA, Netea MG, Van Crevel R. Innate immune recognition of Mycobacterium tuberculosis. Clin Dev Immunol (2011) 2011:405310. doi:10.1155/2011/405310

45. Benard EL, Racz PI, Rougeot J, Nezhinsky AE, Verbeek FJ, Spaink HP, et al. Macrophage-expressed perforins mpeg1 and mpeg1.2 have an anti-bacterial function in zebrafish. J Innate Immun (2015) 7:136-52. doi:10.1159/ 000366103

46. van der Vaart M, van Soest JJ, Spaink HP, Meijer AH. Functional analysis of a zebrafish myd88 mutant identifies key transcriptional components of the innate immune system. Dis Model Mech (2013) 6:841-54. doi:10.1242/ dmm. 010843

47. van der Sar AM, Stockhammer OW, van der Laan C, Spaink HP, Bitter W, Meijer AH. MyD88 innate immune function in a zebrafish embryo infection model. Infect Immun (2006) 74:2436-41. doi:10.1128/IAI.74.4.24362441.2006

48. Elks PM, Brizee S, van der Vaart M, Walmsley SR, van Eeden FJ, Renshaw SA, et al. Hypoxia inducible factor signaling modulates susceptibility to mycobacterial infection via a nitric oxide dependent mechanism. PLoS Pathog (2013) 9:e1003789. doi:10.1371/journal.ppat.1003789

49. Elks PM, van der Vaart M, van Hensbergen V, Schutz E, Redd MJ, Murayama E, et al. Mycobacteria counteract a TLR-mediated nitrosative defense mechanism in a zebrafish infection model. PLoS One (2014) 9:e100928. doi:10.1371/ journal.pone.0100928

50. Deretic V, Saitoh T, Akira S. Autophagy in infection, inflammation and immunity. Nat Rev Immunol (2013) 13:722-37. doi:10.1038/nri3532

51. van der Vaart M, Korbee CJ, Lamers GE, Tengeler AC, Hosseini R, Haks MC, et al. The DNA damage-regulated autophagy modulator DRAM1 links mycobacterial recognition via TLR-MYD88 to authophagic defense. Cell Host Microbe (2014) 15:753-67. doi:10.1016/j.chom.2014.05.005 
52. Hosseini R, Lamers GE, Hodzic Z, Meijer AH, Schaaf MJ, Spaink HP. Correlative light and electron microscopy imaging of autophagy in a zebrafish infection model. Autophagy (2014) 10:1844-57. doi:10.4161/auto.29992

53. Yang CT, Cambier CJ, Davis JM, Hall CJ, Crosier PS, Ramakrishnan L. Neutrophils exert protection in the early tuberculous granuloma by oxidative killing of mycobacteria phagocytosed from infected macrophages. Cell Host Microbe (2012) 12:301-12. doi:10.1016/j.chom.2012.07.009

54. Ramakrishnan L. Looking within the zebrafish to understand the tuberculous granuloma. Adv Exp Med Biol (2013) 783:251-66. doi:10.1007/978-1-4614-6111-1_13

55. Oehlers SH, Cronan MR, Scott NR, Thomas MI, Okuda KS, Walton EM, et al. Interception of host angiogenic signalling limits mycobacterial growth. Nature (2015) 517:612-5. doi:10.1038/nature13967

56. Houben D, Demangel C, van Ingen J, Perez J, Baldeon L, Abdallah AM, et al. ESX-1-mediated translocation to the cytosol controls virulence of mycobacteria. Cell Microbiol (2012) 14:1287-98. doi:10.1111/j.1462-5822.2012.01799.x

57. Cambier CJ, Takaki KK, Larson RP, Hernandez RE, Tobin DM, Urdahl KB, et al. Mycobacteria manipulate macrophage recruitment through coordinated use of membrane lipids. Nature (2014) 505:218-22. doi:10.1038/nature12799

58. Kaufman CK, White RM, Zon L. Chemical genetic screening in the zebrafish embryo. Nat Protoc (2009) 4:1422-32. doi:10.1038/nprot.2009.144

59. Takaki K, Davis JM, Winglee K, Ramakrishnan L. Evaluation of the pathogenesis and treatment of Mycobacterium marinum infection in zebrafish. Nat Protoc (2013) 8:1114-24. doi:10.1038/nprot.2013.068

60. Adams KN, Takaki K, Connolly LE, Wiedenhoft H, Winglee K, Humbert O, et al. Drug tolerance in replicating mycobacteria mediated by a macrophage-induced efflux mechanism. Cell (2011) 145:39-53. doi:10.1016/j. cell.2011.02.022

61. Adams KN, Szumowski JD, Ramakrishnan L. Verapamil, and its metabolite norverapamil, inhibit macrophage-induced, bacterial efflux pump-mediated tolerance to multiple anti-tubercular drugs. J Infect Dis (2014) 210:456-66. doi:10.1093/infdis/jiu095

62. Torraca V, Cui C, Boland R, Bebelman JP, van der Sar AM, Smit MJ, et al. The CXCR3-CXCL11 signaling axis mediates macrophage recruitment and dissemination of mycobacterial infection. Dis Model Mech (2015) 8:253-69. doi:10.1242/dmm.017756

63. Tobin DM, Roca FJ, Oh SF, McFarland R, Vickery TW, Ray JP, et al. Host genotype-specific therapies can optimize the inflammatory response to mycobacterial infections. Cell (2012) 148:434-46. doi:10.1016/j.cell.2011.12.023

64. Roca FJ, Ramakrishnan L. TNF dually mediates resistance and susceptibility to mycobacteria via mitochondrial reactive oxygen species. Cell (2013) 153:521-34. doi:10.1016/j.cell.2013.03.022

65. Kaufmann SH, Lange C, Rao M, Balaji KN, Lotze M, Schito M, et al. Progress in tuberculosis vaccine development and host-directed therapies - a state of the art review. Lancet Respir Med (2014) 2:301-20. doi:10.1016/ S2213-2600(14)70033-5

66. Deng W, Tang X, Hou M, Li C, Xie J. New insights into the pathogenesis of tuberculosis revealed by Mycobacterium marinum: the zebrafish model from the systems biology perspective. Crit Rev Eukaryot Gene Expr (2011) 21:337-45. doi:10.1615/CritRevEukarGeneExpr.v21.i4.40

67. Case ED, Samuel JE. Contrasting lifestyles within the host cell. Microbiol Spectr (2016) 4. doi:10.1128/microbiolspec.VMBF-0014-2015

68. Oksanen KE, Halfpenny NJ, Sherwood E, Harjula SK, Hammaren MM, Ahava MJ, et al. An adult zebrafish model for preclinical tuberculosis vaccine development. Vaccine (2013) 31:5202-9. doi:10.1016/j.vaccine.2013.08.093

69. Ojanen MJ, Turpeinen H, Cordova ZM, Hammaren MM, Harjula SK, Parikka M, et al. The proprotein convertase subtilisin/kexin furinA regulates zebrafish host response against Mycobacterium marinum. Infect Immun (2015) 83:1431-42. doi:10.1128/IAI.03135-14

70. Hammaren MM, Oksanen KE, Nisula HM, Luukinen BV, Pesu M, Ramet M, et al. Adequate Th2-type response associates with restricted bacterial growth in latent mycobacterial infection of zebrafish. PLoS Pathog (2014) 10:e1004190. doi:10.1371/journal.ppat.1004190

71. van Meijgaarden KE, Haks MC, Caccamo N, Dieli F, Ottenhoff TH, Joosten SA. Human CD8+ T-cells recognizing peptides from Mycobacterium tuberculosis (Mtb) presented by HLA-E have an unorthodox Th2-like, multifunctional, Mtb inhibitory phenotype and represent a novel human T-cell subset. PLoS Pathog (2015) 11:e1004671. doi:10.1371/journal.ppat.1004671
72. van der Sar AM, Abdallah AM, Sparrius M, Reinders E, VandenbrouckeGrauls CM, Bitter W. Mycobacterium marinum strains can be divided into two distinct types based on genetic diversity and virulence. Infect Immun (2004) 72:6306-12. doi:10.1128/IAI.72.11.6306-6312.2004

73. Oksanen KE, Myllymäki H, Ahava MJ, Mäkinen L, Parikka M, Rämet M. DNA vaccination boosts Bacillus Calmette-Guérin protection against mycobacterial infection in zebrafish. Dev Comp Immunol (2016) 54:89-96. doi:10.1016/j. dci.2015.09.001

74. Cui Z, Samuel-Shaker D, Watral V, Kent ML. Attenuated Mycobacterium marinum protects zebrafish against mycobacteriosis. J Fish Dis (2010) 33:371-5. doi:10.1111/j.1365-2761.2009.01115.x

75. Stoop EJ, Schipper T, Rosendahl Huber SK, Nezhinsky AE, Verbeek FJ, Gurcha SS, et al. Zebrafish embryo screen for mycobacterial genes involved in the initiation of granuloma formation reveals a newly identified ESX-1 component. Dis Model Mech (2011) 4:526-36. doi:10.1242/dmm.006676

76. Volkman HE, Clay H, Beery D, Chang JC, Sherman DR, Ramakrishnan L. Tuberculous granuloma formation is enhanced by a mycobacterium virulence determinant. PLoS Biol (2004) 2:e367. doi:10.1371/journal.pbio.0020367

77. Knudsen NP, Norskov-Lauritsen S, Dolganov GM, Schoolnik GK, Lindenstrom T, Andersen $\mathrm{P}$, et al. Tuberculosis vaccine with high predicted population coverage and compatibility with modern diagnostics. Proc Natl Acad Sci U S A (2014) 111:1096-101. doi:10.1073/pnas.1314973111

78. Hoang T, Aagaard C, Dietrich J, Cassidy JP, Dolganov G, Schoolnik GK, et al. ESAT-6 (EsxA) and TB10.4 (EsxH) based vaccines for pre- and post-exposure tuberculosis vaccination. PLoS One (2013) 8:e80579. doi:10.1371/journal. pone.0080579

79. Bottai D, Frigui W, Clark S, Rayner E, Zelmer A, Andreu N, et al. Increased protective efficacy of recombinant BCG strains expressing virulenceneutral proteins of the ESX-1 secretion system. Vaccine (2015) 33:2710-8. doi:10.1016/j.vaccine.2015.03.083

80. Lin PL, Dietrich J, Tan E, Abalos RM, Burgos J, Bigbee C, et al. The multistage vaccine $\mathrm{H} 56$ boosts the effects of BCG to protect cynomolgus macaques against active tuberculosis and reactivation of latent $M y$ cobacterium tuberculosis infection. J Clin Invest (2012) 122:303-14. doi:10.1172/JCI46252

81. Dietrich J, Andersen C, Rappuoli R, Doherty TM, Jensen CG, Andersen P. Mucosal administration of Ag85B-ESAT-6 protects against infection with Mycobacterium tuberculosis and boosts prior bacillus CalmetteGuerin immunity. J Immunol (2006) 177:6353-60. doi:10.4049/jimmunol. 177.9.6353

82. Yuan W, Dong N, Zhang L, Liu J, Lin S, Xiang Z, et al. Immunogenicity and protective efficacy of a tuberculosis DNA vaccine expressing a fusion protein of Ag85B-Esat6-HspX in mice. Vaccine (2012) 30:2490-7. doi:10.1016/j. vaccine.2011.06.029

83. Capuano SVI, Croix DA, Pawar S, Zinovik A, Myers A, Lin PL, et al. Experimental Mycobacterium tuberculosis infection of cynomolgus macaques closely resembles the various manifestations of human $M$. tuberculosis infection. Infect Immun (2003) 71:5831-44. doi:10.1128/ IAI.71.10.5831-5844.2003

84. Murray KN, Bauer J, Tallen A, Matthews JL, Westerfield M, Varga ZM. Characterization and management of asymptomatic mycobacterium infections at the Zebrafish International Resource Center. J Am Assoc Lab Anim Sci (2011) 50:675-9.

85. Pasnik DJ, Smith SA. Immunogenic and protective effects of a DNA vaccine for Mycobacterium marinum in fish. Vet Immunol Immunopathol (2005) 103:195-206. doi:10.1016/j.vetimm.2004.08.017

86. Kato G, Kondo H, Aoki T, Hirono I. BCG vaccine confers adaptive immunity against Mycobacterium sp. infection in fish. Dev Comp Immunol (2010) 34:133-40. doi:10.1016/j.dci.2009.08.013

87. Ernst JD. The immunological life cycle of tuberculosis. Nat Rev Immunol (2012) 12:581-91. doi:10.1038/nri3259

88. Berry MP, Graham CM, McNab FW, Xu Z, Bloch SA, Oni T, et al. An interferon-inducible neutrophil-driven blood transcriptional signature in human tuberculosis. Nature (2010) 466:973-7. doi:10.1038/nature09247

89. Kaufmann SH, Dorhoi A. Inflammation in tuberculosis: interactions, imbalances and interventions. Curr Opin Immunol (2013) 25:441-9. doi:10.1016/j. coi.2013.05.005

90. Fletcher HA. Profiling the host immune response to tuberculosis vaccines. Vaccine (2015) 33:5313-5. doi:10.1016/j.vaccine.2015.07.090 
91. Tameris MD, Hatherill M, Landry BS, Scriba TJ, Snowden MA, Lockhart S, et al. Safety and efficacy of MVA85A, a new tuberculosis vaccine, in infants previously vaccinated with BCG: a randomised, placebo-controlled phase 2 b trial. Lancet (2013) 381:1021-8. doi:10.1016/ S0140-6736(13)60177-4

92. Kagina BM, Abel B, Scriba TJ, Hughes EJ, Keyser A, Soares A, et al. Specific $\mathrm{T}$ cell frequency and cytokine expression profile do not correlate with protection against tuberculosis after Bacillus Calmette-Guérin vaccination of newborns. Am J Respir Crit Care Med (2010) 182:1073. doi:10.1164/ rccm.201003-0334OC
Conflict of Interest Statement: The authors declare that the research was conducted in the absence of any commercial or financial relationships that could be construed as a potential conflict of interest.

Copyright $\odot 2016$ Myllymäki, Bäuerlein and Rämet. This is an open-access article distributed under the terms of the Creative Commons Attribution License (CC BY). The use, distribution or reproduction in other forums is permitted, provided the original author(s) or licensor are credited and that the original publication in this journal is cited, in accordance with accepted academic practice. No use, distribution or reproduction is permitted which does not comply with these terms. 